\title{
Die mexikanischen Jahre von Anna Seghers
}

\author{
Douglas Hilt
}

Anna Seghers hat ihre Autobiographie nie geschrieben. Die Gründe dafür bleiben reine Vermutung: ihre ständige Besorgnis eher für andere als für sich selbst, der hektische Zeitplan, auf dem sie bestand, oder schließlich das Gefühl, daß ihr Gesamtwerk - Romane, Novellen, ausgedehnter Briefwechsel und Aufsätze, Interviews mit der Presse und in Radio und Fernsehen - genügend aussagte. Die bewegten Exiljahre, zuerst in Frankreich und später in Mexiko, dann die Nachkriegsjahre in Ostdeutschland, haben ihr einfach nicht die Zeit gelassen, an jener eher introspektiven Übung teilzunehmen. Infolgedessen bleiben viele Episoden in ihrem Leben ungeklärt, besonders die Kriegsjahre im mexikanischen Exil.

Im Juli 1937, mitten im spanischen Bürgerkrieg, besuchte Anna Seghers den Zweiten Internationalen Schriftstellerkongress in Madrid. Sie wurde von ihrem Freund Bodo Uhse begleitet, einem ehemaligen Nazi, der seinen Irrtum widerrufen hatte und jetzt, genau so wie sie, sich als erklärter Kommunist verstand. Seghers hatte auch die Bekanntschaft Ludwig Renns gemacht, des sächsischen Schriftstellers, der aktiv auf der Seite der Republikaner kämpfte. Sowohl Uhse als auch Renn, zusammen mit anderen deutschen Veteranen des Bürgerkrieges, fanden später Zuflucht in Mexiko.

In ihrem Roman Transit hat Seghers die Verwirrung und die chaotische Flucht durch Südfrankreich nach dem Einmarsch der Nationalsozialisten im Sommer 1940 plastisch dargestellt. Obwohl die Kritik gewarnt hat, den Roman als Autobiographie zu betrachten (Batt 1973, 155), ist Transit trotzdem das persönlichste und auch das realistischste aller ihrer Werke. Die lebhaften Szenen im mexikanischen Konsulat, die "tödliche Bürokratie", auch dann wenn die Flüchtlinge zwischen Leben und Tod schweben, die allerletzte Hoffnung, alles wird peinlich genau geschildert. Das mexikanische Visum hat Anna Seghers und ihren ungarischen Ehemann, genau so wie viele andere Emigranten, sehr wahrscheinlich vor dem sicheren Tod gerettet. Hans Albert Walter formulierte es folgendermaßen: "Hätte ihnen nicht Mexiko Asyl gewährt, so wären sie in Frankreich schutzlos dem Zugriff der Gestapo ausgeliefert gewesen" $(1973,35)$.

Auch so ist es ihnen nur knapp gelungen, an Bord des Frachters Paul Lemerle den Hafen von Marseille am 24. März $1941 \mathrm{zu}$ verlassen. Nach einer mehrwöchigen Internierung auf der Insel Martinique ging die Odyssee über die Dominikanische Republik und Ellis Island weiter, bis Seghers, ihr Mann und ihre zwei Kinder schließlich in Veracruz nach einer Seereise landeten, die so lange dauerte wie diejenige von Kolumbus im späten XV. Jahrhundert. Kein Wunder, daß Seghers so oft den mexikanischen Präsidenten Lázaro Cárdenas lobte, der während seiner Amtszeit die Veteranen des spanischen Bürgerkrieges 
und auch andere gleichgesinnte Kämpfer gegen den Faschismus ermutigte, um Asyl in Mexiko nachzusuchen.

Gleich am Anfang spürte Seghers den Geldmangel. Sie hatte manche Schwierigkeiten zu überwinden, um Geld zu verdienen und für ihre Familie sorgen zu können. Trotzdem schrieb sie am 12. Juli 1941, bald nachdem sie gelandet war, ihrem Freund Wieland Herzfelde in New York: "Soweit ich hier schon was vom Leben gerochen habe, gefällt es mir sehr. Ich glaube, ich fühle mich hier fast besser als in New York. Ich werde gut arbeiten können" (Seghers/Herzfelde 1986, 43). Im Oktober des gleichen Jahres trug Seghers zur Sammlung von 500 Pesos, damals etwa $\$ 100$, bei, um eine neue politischliterarische Monatsschrift der antifaschistischen "Bewegung Freies Deutschland" zu gründen. Der erste Chefredakteur war Bruno Frei, gefolgt von Alexander Abusch; außer Seghers trugen auch andere Schriftsteller wie ihr Freund Bodo Uhse, André Simone, Ludwig Renn und Egon Erwin Kisch zu diesem literarischen Unternehmen bei.

Einen Monat später, im November, wurde der "Heinrich-Heine-Club" gegründet mit der Absicht, die vielen Zänkereien zu vermeiden, die so oft die Tätigkeiten der älteren "Liga Pro-Cultura Alemana" beeinträchtigt hatten. Bei der ersten Sitzung wurde Seghers von den ungefähr hundert Anwesenden zur Präsidentin der neuen Organisation ernannt. Zwei Wochen später, am 21. November, las Seghers zwei Kapitel aus ihrem noch unveröffentlichten Roman Das siebte Kreuz vor, der nach einem erregten Briefwechsel und manchen Mißverständnissen gerade vom amerikanischen Verlag Little, Brown and Company akzeptiert worden war.

Wie Das siebte Kreuz überhaupt herausgebracht wurde, war beinahe so kompliziert wie die Seereise der Autorin zur Neuen Welt. Seghers hatte die ersten Kapitel in Paris schon 1938 angefangen, und eine Zeitlang dachte sie, diese seien während der Flucht durch Frankreich im Sommer 1940 verlorengegangen. Aber dank dem Weitblick ihres Freundes Franz Weiskopf wurde eine von zwei Abschriften gerettet und nach Amerika gebracht. Durch die Hilfe Erich Maria Remarques wurde der Roman von James Galston ins Englische übersetzt, der manchmal ganze politische Passagen abmilderte, die er als "langweilig" bezeichnete. Dazu ließ er andere Sätze ganz einfach weg, zum großen Bedauern der Verfasserin, die nicht imstande war, etwas dagegen zu tun. Sehr wahrscheinlich war es die Tatsache, daß der Protagonist Georg Heisler in der Übertragung nicht mehr als Kommunist erscheint, die es ermöglichte, den Roman für den amerikanischen Leser attraktiv zu machen und später mit Spencer Tracy in der Hauptrolle zu verfilmen. ${ }^{1}$

Als "Book of the Month" hatte The Seventh Cross einen Riesenerfolg. 319.000 Exemplare wurden in den ersten zwölf Tagen verkauft, und im Febru-

${ }^{1}$ Eine ausführliche Darstellung erscheint in den zwei Aufsätzen von Alexander Stephan, 1985 und 1988. 
ar 1943, als MGM sich entschied den Film zu drehen, stieg die Zahl bis auf 421.000 an. Wie im Falle der amerikanischen Fassung des Romans war Seghers weder vom Drehbuch noch vom Film begeistert. Laut der beiden Verträge sollten ihr etwa $\$ 300.000$ bezahlt werden, tatsächlich aber reduzierte sich diese Summe erheblich sowohl durch Bezahlungen an verschiedene Agenten als auch durch die Tatsache, daß sie im Ausland als "enemy alien" und noch dazu als ungarische Staatsbürgerin betrachtet und behandelt wurde.

Der große Erfolg des Romans The Seventh Cross ist nicht schwer zu erklären. Das Antinazi-Thema wurde in den Hintergrund gedrängt, und sowohl der Roman als auch der Film wurden hauptsächlich als Thriller behandelt, eine gewissermaßen vom FBI inszenierte europäische Verbrecherjagd. Anna Seghers' Botschaft, daß es trotz aller Nazi-Greueltaten "noch gute Deutsche gebe", blieb jedoch erhalten. Die wenigsten Amerikaner aber wußten, daß die Autorin eine zur Zeit in Mexiko wohnhafte Deutsche war, und noch weniger war ihnen bekannt, daß ihre eigene Regierung sie aus dem "Land of the free" verbannt hatte.

Die ursprüngliche deutsche Fassung des Romans hatte es noch schwerer. Wie alle deutschen Schriftsteller in Mexiko verlor auch Seghers beinahe ihre ganze Leserschaft. In den frühen dreißiger Jahren wohnten etwa 6500 Deutsche in Mexiko, davon etwa die Hälfte in der Hauptstadt. ${ }^{2}$ Gegen 1942 hatte diese Anzahl durch die Ankunft von etwa 1600 jüdischen Flüchtlingen beträchtlich zugenommen. Obgleich diese neue Gruppe kulturell deutsch, nicht zionistisch eingestellt und im allgemeinen nicht besonders religiös war, bekam sie trotzdem die Feindseligkeit der Mehrheit in der älteren etablierten deutschen Kolonie zu spüren. Eine Gruppe Exilanten entschied sich, ihren eigenen Verlag El libro libre ("Das freie Buch") zu gründen. Das siebte Kreuz wurde dessen dritter Titel; die Gesamtausgabe von etwa 5000 Bänden machte aber nur weniger als ein Hundertstel der amerikanischen Version aus.

Am 24. Juni erlitt Anna Seghers sehr schwere Kopfverletzungen, als sie von einem vorbeirasenden Wagen auf dem Paseo de la Reforma in der Stadtmitte von Mexiko überfahren wurde. In einem späteren Brief, der den 50. Geburtstag Seghers' feierte, beschrieb ihr Freund Bodo Uhse seine Reaktion:

Ich weiß nicht mehr, wer uns die bös erschreckende Nachricht brachte, daß $\mathrm{Du}$ - während wir so geredet - mit zertrümmerter Schädeldecke auf der regenüberwaschenen Prachtstraße Mexikos, dem Paseo de la Reforma, gelegen hattest, bewußtlos und für Stunden ohne Hilfe. Ein vorbeirasendes Auto hatte Dich umgerissen, als Du die Straße überqueren wolltest, und nie ist geklärt worden, ob ein leichtfertiges Unglück oder wohlüberlegte mörderische Absicht dabei das Steuer geführt hatte. Nicht Tage, sondern Wochen schwanktest Du zwischen Leben und Tod (Seghers/Herzfelde 1986, 195).

${ }^{2}$ von zur Mühlen 1988, 164. S. auch Pohle 1986, 24. 
Der Vorfall ist niemals befriedigend aufgeklärt worden. Trotz ihrer schweren Verletzungen konnte sie am 27. Juli den Filmvertrag mit MGM unterzeichnen und war so weit genesen, daß sie am 1. November an Herzfelde schrieb: "Wie Du siehst, bin ich wiederhergestellt. Es geht mir noch ganz gut. Meine Augennerven sind noch verletzt, doch ich schreibe wieder Briefe, ja sogar Novellen, noch sehr langsam, aber ich tue es" (ebd., 55).

Während ihrer Genesung dachte Seghers charakteristischerweise bereits an Zukunftspläne. Am 17. Dezember teilte sie Herzfelde mit, daß sie eine neue Novelle angefangen hatte: "Über die Geschichte, die ich erwähnte, Folgendes: Ihr Titel: Der Ausflug der toten Mädchen. Umfang: 30 bis 40 Seiten Schreibmaschine" (ebd., 58). Das Thema ist die ferne Erinnerung an einen Schulausflug in Mainz kurz vor Ausbruch des Ersten Weltkrieges. Das Ganze wird sehr poetisch durch den Filter des mexikanischen Exils wachgerufen, wobei die sanfte Landschaft des Rheintals neben die grelle Kargheit des mexikanischen Hochlands gestellt wird. In einem einsichtigen Aufsatz bemerkt Kathleen LaBahn $(1986,74)$ Folgendes über Seghers Schilderung: "The intertwining of literary intent and historical reality is so flawless that it is essentially impossible to single out either aspect for discussion".

Von allen Werken Seghers' lag Der Ausflug der toten Mädchen der Autorin am nächsten. Sie war durchaus mit dem mexikanischen Glauben vertraut, daß die Toten persönlich an Familienfesten teilnehmen, so am "día de los muertos", wo die neu Verstorbenen das Abendbrot mit den Lebendigen teilen. Auch kannte sie die berühmte Freskomalerei "Sonntagsträumerei in der Alameda" von Diego Rivera, welche die Gegenwart eines grinsenden Skeletts in bunten Farben darstellt. Aber die treibende Kraft für die Geschichte war sicher die Nachricht von der Zerstörung ihrer Heimatstadt Mainz durch einen schweren Luftangriff, die im Roman lebhafte Kindheitserinnerungen mit dem tragischen Schicksal jener unschuldigen Ausflüglerinnen verknüpfte. Die Ereignisse werden in der ersten Person erzählt, und obgleich die Handlung - das Schicksal der einzelnen Schulmädchen - fiktiv ist, ist dieses Seghers' einziges Werk, in dem das Autobiographische durch den ganzen Text schimmert, auch wenn die genauen Details unterdrückt sind.

Die Geschichte beginnt zunächst schlicht. Die Erzählerin befindet sich in einer Pulquería - warum wird nicht erklärt - , einer Kneipe am Stadtrand eines sonnenüberfluteten, staubigen mexikanischen Dorfes. Der Wirt möchte natürlich wissen, woher diese Fremde komme und nimmt an, sie müsse eine Gringa von nördlich der Landesgrenze sein. Aber auf seine Frage antwortet sie: "Nein, von viel weiter her. Aus Europa" $(1964,207)$. Ihre spontane Antwort überrascht sie genauso sehr wie ihren mißtrauischen Wirt, denn sie setzt eine Reihe von Betrachtungen über ihr Leben in einem solchen fremden und exotischen Land in Gang, über die Veränderungen, die sie erlebt hat. Die ganze Frage ihrer Vergangenheit, Gegenwart und Zukunft wird zu einer Bewertung der Existenz und der Realität, Themen, die alle Exilanten berühren. 
Seghers kann die trockene, heiße, öde mexikanische Landschaft niemals als Heimat betrachten: "Um Rettung genannt zu werden, dafür war die Zuflucht in diesem Land zu fragwürdig und zu ungewiß. Ich hatte Monate der Krankheit gerade hinter mir, die mich hier ereicht hatte, obwohl mir die mannigfachen Gefahren des Krieges nichts hatten anhaben können" (ebd.). Offensichtlich ist die Erzählerin sowohl körperlich wie geistig vollkommen erschöpft, aber trotz ihrer Müdigkeit muß sie weiterziehen: "Es gab nur noch eine einzige Unternehmung, die mich anspornen konnte: die Heimfahrt" (208). Für sie ist das vorläufige Heim "das Rancho", durch einen Kaktuszaun isoliert und von einem räudigen Köter verteidigt. Seghers betritt das heruntergekommene Gebäude sehr vorsichtig und vernimmt den regelmäßigen Rhythmus einer Kinderschaukel. Im gleichen Moment ruft eine Stimme sie "Netty!" - der Jugendname, den die Autorin seit zwanzig Jahren nicht mehr gebraucht hat. Mit einem Schlag wird die Kluft zwischen ihrem Exil in einem fernen exotischen Land und den bewegten Jahren, die jenem sorglosen Schulausflug am Rhein folgten, überbrückt:

Mit diesem Namen hatte mich seit der Schulzeit niemand mehr gerufen. Ich hatte gelernt, auf alle die guten und bösen Namen zu hören, mit denen mich Freunde und Feinde zu rufen pflegten, die Namen, die man mir in vielen Jahren in Straßen, Versammlungen, Festen, nächtlichen Zimmern, Polizeiverhören, Büchertiteln, Zeitungsberichten, Protokollen und Pässen beigelegt hatte (ebd., 209).

Als sie noch im Spitalbett lag, während sie oft in Bewußtlosigkeit versank, dachte Anna manchmal an ihren früheren Namen, in der Hoffnung, dadurch eine magische Genesung hervorzuzaubern. Anna - oder besser gesagt: Netty - ist dies vielleicht zu gut gelungen: Der ausgedörrte Kaktus und die versengende erbarmungslose Hitze des mexikanischen Hochlands werden durch das sanfte üppige Wachstum des dicken Grases, durch die lauen Brisen, die vertrauten Blumen und weichen Wolken ihrer sorglosen Schulmädchenjahre ersetzt.

Doch ist diese beruhigende Szene durchaus trügerisch. Wie es so oft der Fall bei Exilanten ist, werden Zeitbegriff und Erinnerung hoffnungslos durcheinandergebracht und Einzelheiten in verworrenen Bruchstücken ineinandergeschoben. Die Gesichter und Besonderheiten sowohl ihrer Schulkameradinnen wie auch ihrer Lehrerinnen werden in Nettys Erzählung aus einer längst vergangenen Epoche in die Gegenwart zurückgerufen und ihre Karrieren und Schicksale in einem oder zwei aufschlußreichen Sätzen skizziert. Versprechen werden gemacht und gebrochen. Einige von Nettys Schulkameradinnen verrieten in späteren Jahren ohne Gewissensbisse ihre jetzt ältere jüdische Lehrerin. Eine heiratete einen hohen Nazibeamten, während zwei andere, die nach außen hin am wenigsten Mut zeigten, trotzdem später die innere Stärke fanden, sich der Barbarei des Nazistaates soweit zu widersetzen, daß sie ihr eigenes Leben aufopferten. Längst ist die mexikanische Dürre verblaßt, und die Schilderung schwankt jetzt zwischen dem wehmütigen Ausflug ins Restaurant am 
Rheinufer und der späteren tragischen Zerstörung der Stadt Mainz und dem damit verbundenen schrecklichen Tod von Nettys Freundin Marianne. Dieses Ereignis konnte selbstverständlich - von der Autorin im Zwangsexil eine halbe Welt entfernt - nur aus der Vorstellung dargestellt werden, aber das Wachrufen unerlebter und trotzdem stark empfundener Ereignisse klingt hier genauso wahr wie der genaueste Augenzeugenbericht.

In der Phantasie von Seghers wird - in ihrer liebevollen Beschreibung der Rückkehr zum Familienheim, nachdem sie den ganzen Tag auf einem Ausflug auf dem Land verbracht hatte, - Mainz sehr poetisch dargestellt. Unwichtige Einzelheiten, an sich ohne Bedeutung, machen das Wesentliche des Exilantengedächtnisses aus: Blumenbeete voll Geranien und Begonien in den Anlagen, die Bogen der Rheinbrücken, die karierten Tischtücher in den Restaurants, der Dunst, der aus dem Fluß aufsteigt. Als die Erzählerin die Treppen zum Familienheim und zur zärtlichen Umarmung der Mutter hinaufeilt, kann sie schon das Geschirrklappern in der Küche bei der Vorbereitung des Abendessens vernehmen. Erst jetzt wird ihr klar, daß das, was sie gehört hat, das rhythmische Händeklatschen bei der Zubereitung von Tortillas gewesen war, vermischt mit dem kakophonen Gackern der Truthähne im staubigen Hinterhof. Die grelle Sonne brennt immer noch hoch über dem Horizont, der räudige Köter liegt am gewohnten Platz, der Wirt hat sich nicht bewegt. Damit ist sie unmißverständlich nach Mexiko und zur Realität zurückgekehrt.

Für die Mehrzahl der deutschen Exilanten in Mexiko war das tägliche Leben oft durch bittere Rivalitäten, vielfach politischer Natur, gekennzeichnet. Gerade in dem Moment, als der neue "Heinrich-Heine-Klub", dessen Präsidentin Anna Seghers war, und der Verlag "El libro libre" einen bescheidenen Erfolg genossen, wurde die ältere, im Jahre 1937 gegründete "Liga Pro-Cultura Alemana" das Opfer ihrer eigenen inneren Meinungsverschiedenheiten. Gehässige persönliche Angriffe waren keineswegs ungewöhnlich. Gustav Regler, der während des spanischen Bürgerkriegs schwer verwundet worden und mit seinen ehemaligen Kameraden bitter zerstritten war, bangte ständig um sein Leben und war mit einem Revolver bewaffnet. In dieser vergifteten Atmosphäre setzte sich Seghers für Mäßigung und Zusammenarbeit ein. Andere Exilanten glaubten tatsächlich, sie würden eine führende Rolle im Deutschland der Nachkriegszeit spielen. Man fühlte sich sogar bemüßigt, eine deutsche Exilregierung zu bilden. In einem solchen Kabinett wurde Seghers zur Kulturministerin ernannt. Als sich das Kriegsende näherte und das Ausmaß der nationalsozialistischen Greueltaten offensichtlich wurde, nahm eine ziemlich große Anzahl von Exilanten eine deutschfeindliche Haltung ein. Sie legten eine gewisse "Versailler Mentalität" an den Tag, indem die Bestrafung einer ganzen Nation gefordert wurde. Seghers, nebst anderen Gemäßigten, warnte vor einer solchen Verdammung und bezeichnete jene Gedanken als eine Art Rassismus.

Endlich war der Krieg in Europa beendet. Anna Seghers hatte nie daran gezweifelt, daß sie eines Tages nach Deutschland zurückkehren würde. Es fragte sich nur, in welches Deutschland? Bevor sie von Mexiko Abschied nahm, 
hatte sie schon eine andere Novelle mit dem Titel Die Toten bleiben jung begonnen. Am 22. April $1947 \mathrm{kam}$ sie in Ostberlin an. Dort fand sie die politische Atmosphäre angenehmer als in ihrer Heimatstadt Mainz. Mit ihrer üblichen Energie und Überzeugung warf sie sich in ihr neues Leben. Doch die Jahre in Mexiko vergaß sie nie.

Schon 1950, d.h. nach der Berliner Luftbrücke, schrieb Seghers eine ihrer liebenswertesten Novellen, die ergreifende Erzählung von Crisanta, einem armen sechzehnjährigen Waisenmädchen, das sein Heimatdorf Pachuca verläßt, um ein besseres Leben in der Hauptstadt zu finden. Das tragische Ergebnis verlorener Hoffnungen und Verführung ist beinahe unvermeidlich. Offensichtlich besteht die Originalität der Erzählung keineswegs in der Darstellung der Ereignisse und Gefühle, auch wenn sie sehr genau beschrieben sind. Auch ist ihre hervorragende Qualität nicht mit irgendeiner Philosophie oder einem sozialen Ausdruck verbunden. Sie enthält eher eine allgemeine Kritik des durch Außenseiter bewirkten Eindringens entmenschlichter Maschinen: "Sie sagten mir dort, kurz vor mir sei eine neue Maschine angekommen... ein Gringo". $(1988,19)$. Die Wirkung der Erzählung beruht im wesentlichen auf der Wärme des Symbolismus, wie z. B. in "das unvergleichliche, unbegreifliche tiefe und dunkle Blau" des Rebozo, des Schals, der gleichzeitig schützt und auch viel verbirgt, der durch die Jahrhunderte ein Gefühl von Geborgenheit geschaffen hat, so daß das Volk hat überleben und sich sogar durchsetzen können. Kurt Batt bemerkt dazu: "Das Gefühl des Verwachsenseins mit der Heimat und der Geborgenheit in Familie, Gemeinde und Volk, wie sie es bei ihren Begegnungen mit den einfachen Mexikanern erlebte, mag die exilierte Schriftstellerin gerührt und betroffen gemacht haben". $(1973,175)$. Die tragischen Vorfälle enthalten einen implizierten Vorwurf des Zusammenbruchs der traditionellen Familie und zeigen den Auszug der arglosen Campesinos, die ihr Glück in der gefühllosen berechnenden Stadt suchen.

In der märchenhaften Atmosphäre der Busfahrt zur Hauptstadt verliebt sich Crisanta in Miguel. Um eine bessere Zukunft für sie beide zu sichern, immatrikuliert sie sich an der Abendschule, damit sie wenigstens lesen und schreiben kann und dadurch nicht ihr ganzes Leben in der Tortillafabrik ihrer Tante verbringen muß. Aber gute Absichten und harte Arbeit genügen nicht; Miguel verläßt sie, und aus lauter Verzweiflung flieht Crisanta in die kalte Nacht der herzlosen Stadt, wird Strichmädchen und ist bald in anderen Umständen. Es folgen eine Abtreibung und eine zweite Schwangerschaft, und nur mittels der Rückkehr in ihr Heimatdorf, wo sie in ihrem blauen Rebozo Früchte verkauft, gelingt es ihr, Erlösung zu finden. Die Erzählung enthält keine Sentimentalität, kein Selbstmitleid oder Bedauern. Das Leben des mexikanischen Alltags ist genauso erbarmungslos wie das Klima, und für die überwiegende Mehrheit der Menschen gibt es kaum einen sozialen Fortschritt von einer Generation zur nächsten, nur die gleiche Wiederholung von drückender Armut und Verzweiflung. Doch durch dieses unveränderliche Elend hindurch spürt man einen 
dünnen Faden Hoffnung, eine gewisse Stärke und Entschlossenheit, die stillschweigend von einer Generation zu nächsten weitergegeben wird.

Im Laufe der Jahre kehrte Seghers in Gedanken oft nach Mexiko zurück. Als junge Kunsthistorikerin kurz nach dem Ersten Weltkrieg hatte sie ihre Doktorarbeit über Rembrandt geschrieben, und während ihres ganzen Lebens war sie davon überzeugt, daß die Kunst von schöpferischen Kräften durchdrungen sei. Keiner, der die revolutionären Fresken von Diego Rivera, José Clemente Orozco oder David Siquieros an den Wänden und Decken zahlloser Gebäude in Mexiko gesehen hat, bleibt unbeeindruckt von ihrer elementaren Kraft und dem Gebrauch strahlender Farben. Diese Erinnerungen spiegeln sich in zwei Aufsätzen wider, die Seghers über mexikanische Wandgemälde schrieb, nämlich Die gemalte Zeit und Diego Riveras Fresken. Szenen präkolumbianischer idyllischer Volkstänze und Erntefeste werden bei ihr neben Darstellungen der Grausamkeit des spanischen Kolonialismus und des späteren, von gefühllosen Yankee-Geschäftsleuten einem darniederliegenden Mexiko aufgezwungenen Industrialismus gestellt. In diesen Aufsätzen wird die Verbindung von Seghers' künstlerischen Gefühlen und ihrem tiefen Sozialbewußtsein deutlich.

In einem Interview mit Wilhelm Girmus aus dem Jahre 1967 dachte Seghers über ihre Exiljahre in Mexiko nach: "Es hat mir dort außerordentlich gut gefallen, und ich habe in diesem Land viel gelernt und sehne mich, noch einmal hinzukommen" (Batt 1973, 173). Ihr Wunsch ist nie in Erfüllung gegangen, aber noch im gleichen Jahr des Interviews wurde ihre Vision Mexikos in die kurze Erzählung Das wirkliche Blau, die persönliche Suche nach der eigenen wahren künstlerischen Seele, übertragen. Die Geschichte handelt von der Odyssee eines begabten Töpfers namens Benito Guerrero, der infolge des Krieges in Europa die einzigartigen dunkelblauen Farbstoffe aus Deutschland, die sein populäres feines Tafelgeschirr kennzeichnen, nicht mehr erhalten kann. Ohne Zweifel sah Anna Seghers, die diese Erzählung im sowjetischen Armenien schrieb und sie "meinen armenischen Freunden, Schriftstellern und Töpfern" widmete, die Parallele zu den Volkskünstlern, die noch verhältnismäßig unverdorben durch das Vordringen des industriellen Zeitalters ihre Natürlichkeit bewahrten.

Benito sieht sich dazu gezwungen, die gleichen unnachahmlichen Farbstoffe von einem entfernten Vetter zu beziehen, der dieselbe magische Farbe aus dem Felsen eines alten Silberbergwerkes gewonnen hat. Aus Gründen, die sich seiner Kontrolle entziehen, muß sich Benito jetzt nach innen wenden, um das Geheimnis des unerreichbaren Blaus im Herzen seines eigenen Volkes zu finden, in dem hohen Gebirge der Sierra Madre, wo das Azur des Himmels und das Dunkelblaue tief in den Adern der Erde ein und dasselbe werden. Für die deutschen Romantiker stellt die Suche nach "einer hohen lichtblauen Blume" das Traumsymbol der Sehnsucht dar; für Benito ist diese Farbe keine entfernte Abstraktion, sondern eher die Würde des einzelnen Arbeiters, der Stolz des schöpferischen Künstlers, der durch seine standhafte Ehrlichkeit auch seine Gemeinde bereichert. 
Solche Geheimnisse offenbaren sich nicht leicht. Im Gegensatz zu vielen ihrer Mitexilanten, die das Leben außerhalb der Hauptstadt als eine Rückkehr in die Barbarei betrachteten, interessierte sich Seghers für das ländliche Leben und wußte wohl die künstlerische Begabung des Volkes zu schätzen. Gerade hier hatte sie das Gefühl, tägliche Arbeit und Kunst seien in einer harmonischen Einheit verbunden. Bei Seghers war dies keineswegs eine sentimentalische Sehnsucht nach irgendeinem vorindustriellen Garten Eden - ihr eigener marxistischer Hintergrund oder vielmehr ihre höchst persönliche schmerzvolle Lebenserfahrung hätten eine solche Einstellung ausgeschlossen -, sondern sie war sich des erbärmlichen Elends nur zu sehr bewußt, das sowohl Das wirkliche Blau als die frühere Crisanta durchdringt. Auf diesen Blättern, die wohl zu ihren schönsten gehören, ist die künstlerische Sensibilität von einem fein entwickelten Sozialbewußsein nicht zu trennen.

Es ist sehr leicht, den Einfluß Mexikos auf Seghers Werk zu übertreiben. Ihren Roman Die Toten bleiben jung hatte sie in Mexiko begonnen, und er wurde in Berlin 1949 herausgegeben. Es handelt sich jedoch dabei beinahe ausschließlich um die Ereignisse in Deutschland zwischen 1918 und der Niederlage der Nationalsozialisten. Fest steht hingegen, daß in ihrer kurzen Erzählung Die Heimkehr des verlorenen Volkes vom Jahre 1965 Präsident Cárdenas als der Befreier der Mayas reinkarniert wird, als Symbol der Freiheit in allen Zeitaltern. Als Kontrast dazu handelt eine andere Erzählung, Post ins gelobte Land, die während 1943/44 geschrieben wurde, d.h. mitten in ihren Jahren in Mexiko, durchaus von einer anderen Epoche und einer anderen, entfernten Welt. Wie so oft bei Exilschriftstellern sind es genau diese Isolierung und die Entfernung von der Heimat, die solch starke Erinnerungen hervorrufen.

Im Januar 1947 erhielt Anna Seghers endlich ein amerikanisches Durchreisevisum, das es ihr ermöglichte, über New York wieder nach Europa zurückzukehren. Ihr Gepäck wog wenig, aber mit sich trug sie unvergeßliche Eindrücke eines exotischen Landes, das sie viel gelehrt hatte und dem sie immer verpflichtet blieb. An manchem kalten, nebligen Morgen in Ostberlin kehrten ihre Gedanken zu jenem fernen Land der brennenden Landschaften und tiefen Gefühle zurück. Mexiko lag eine halbe Welt weit entfernt, in ihrem Herzen aber war es niemals sehr weit weg. Bis zu ihrem Tod blieb Mexiko für Anna Seghers ein Teil ihres Lebens. 


\section{Bibliographie}

Batt, Kurt. 1973. Anna Seghers: Versuch über Entwicklung und Werke. Frankfurt: Röderberg.

LaBahn, Kathleen J. 1986. Anna Seghers' Exile Literature. The Mexican Years (1941-1947). New York/Bern/Frankfurt: Lang.

Pohle, Fritz. 1986. Das mexikanische Exil. Stuttgart: Metzler.

Seghers, Anna. 1964. Der Ausflug der toten Mädchen. Darmstadt: Luchterhand.

-. 1988. Crisanta. Darmstadt: Luchterhand.

Seghers, Anna; Herzfelde, Wieland. 1986. Gewöhnliches und gefährliches Leben. Ein Briefwechsel aus der Zeit des Exils 1939-1946. Darmstadt: Luchterhand.

Stephan, Alexander. 1985. Ein Exilroman als Bestseller. In: Exilforschung. Ein internationales Jahrbuch. III, München: Ed. Text und Kritik.

-. 1988. Anna Seghers' The Seventh Cross. Ein Exilroman über Nazideutschland als Hollywood-Film. In: Ebd., VI, München: Ed. Text und Kritik.

Mühlen, Patrik von zur. 1988. Fluchtziel Lateinamerika. Die deutsche Emigration 1933-1945. Bonn: Neue Gesellschaft.

Walter, Hans Albert. 1973. In: Heist, Walter (Textred.). Anna Seghers aus Mainz. Mainz: Krach. 